\title{
Immunotherapy Combined With Chemotherapy and Radiotherapy for a Patient With Cancer of Unknown Primary: A Case Report
}

\section{Qingtao Ni}

Jiangsu Taizhou People's Hospital

KaiJin Lu

Jiangsu Taizhou People's Hospital

Chi Pan

Jiangsu Taizhou People's Hospital

ShengBin Dai

Jiangsu Taizhou People's Hospital

Peng Wang ( 5655818@qq.com )

Department of Oncology, Jiangsu Taizhou People's Hospital, Taizhou, China

\section{Case Report}

Keywords: cancer of unknown primary, immunotherapy, chemotherapy, radiotherapy, case report

Posted Date: October 13th, 2020

DOl: https://doi.org/10.21203/rs.3.rs-89838/v1

License: (c) (i) This work is licensed under a Creative Commons Attribution 4.0 International License. Read Full License 


\section{Abstract}

\section{Background:}

Cancer of unknown primary (CUP) is metastatic at diagnosis with unknown primary site, indicating a high degree of malignancy with poor prognosis. The development and application of targeted therapy and Immunotherapy is the current research hotspot, and provide more treatment options for CUP.

\section{Case Presentations:}

A 36-year-old freeworking male presented with pain on the right hip in April 2018. After various examinations, he was diagnosed as CUP. He received chemotherapy, immunotherapy and local radiotherapy in our department. However, the use of radiotherapy after immunotherapy resulted in severe pneumonia.

\section{Conclusions:}

Compared with traditional treatments, immunotherapy is effective treatment with fewer side effects and better patient tolerance. However, treating physicians should continue to pay special attention to the occurrence of side effects, when radiotherapy combined with immunotherapy.

\section{Background}

Cancer of unknown primary (CUP) is a mysterious type of cancer. It is metastatic at diagnosis with unknown primary site, indicating a high degree of malignancy with poor prognosis. ${ }^{1}$ In a previous study, CUP was diagnosed as a malignant tumor and the metastasis was confirmed through histology. However, despite a comprehensive diagnostic work-up, the primary site was not determined. ${ }^{2}$ The development and application of targeted therapy and immunotherapy is the current research hotspot, and provide more treatment options for CUP. Recently, a patient with CUP and multiple systemic metastases was treated using immunotherapy combined with chemotherapy and radiotherapy in our department.

\section{Case Presentations}

A 36-year-old freeworking male presented with pain on the right hip in April 2018. Positron emission computed tomography (CT) was performed, demonstrating probable bone metastasis and surrounding soft tissue masses in the pelvis (Figure 1A) and the upper lobe of the right lung (Figure 1B). Emission CT of bone was conducted, which revealed bone metastasis. Pelvic puncture of the soft tissue masses in the posterior superior acetabulum and the iliac crest indicated poorly differentiated mucinous adenocarcinoma. Immunohistochemistry confirmed the presence of mucinous adenocarcinoma, with some cells being signet-ringoid, cytokeratin $7($ CK7) $(+)$, CK19 (+), thyroid transcription factor-1 (-), anaplastic lymphoma kinase-CST (-), CK20 (-), caudal-related homeodomain transcription 2 (CDX2) (-), and Napsin A (-). Pathological consultation at the Fudan University Shanghai Cancer Center indicated 
that the tumors were derived from the digestive tract. However, following gastroscopic and colonoscopic examination, the primary site remained unknown. The patient was subjected to immune-related gene testing. The results showed programmed cell death 1 ligand 1 (PD-L1) positivity (25\%), the tumor mutational burden (10.48\%), and mutation abundance of exon 5 in TP53 (49.49\%). Therefore, the patient was diagnosed with CUP.

In May 2018, the patient received palliative care to alleviate the pain and special conformal radiotherapy with DT40GY/20F for the bone metastasis and surrounding soft tissue mass (Figure 1A). Subsequently (June 2018), he received chemotherapy with "XELOX" regimen (i.e., capecitabine 1,500 $\mathrm{mg}$ in the morning and $2,000 \mathrm{mg}$ in the evening per os $\mathrm{d} 1-14+$ oxaliplatin $130 \mathrm{mg} / \mathrm{m}^{2}$ intravenous $\mathrm{d} 1$ ). The selection of the regimen was based on recommendations pertaining to first-line chemotherapy for the treatment of advanced tumors of the digestive tract.

During the treatment period, the pain in the left ilium intensified. Conformal radiotherapy with DT40GY/20F to the site was initiated on June 15, 2018. The size of the mass on the chest wall was $15 \times 15 \mathrm{~mm}$ (Figure 1C), and the mass in the lung was enlarged (Figure 1D). These findings indicted progressive disease. A biopsy for the mass of the chest wall was performed to identify the primary lesion. Based on the morphology with H\&E staining, $\times 40$ (Figures 2A) and H\&E staining, $\times 100$ (Figures 2B) and combined with the immunohistochemistry results, the pathological analysis indicated the presence of poorly differentiated mucinous adenocarcinoma, solid type (signet-ring cell), immunohistochemistry results can exclude mesothelial and mammary gland sources. According to clinical endoscopy and other tests, we excluded the digestive tract as the source of the cancer. It was considered that the tumor originated in the lung. Subsequently, the patient received second-line chemotherapy (paclitaxel $200 \mathrm{mg}$ d1 $1-8+$ cisplatin 30 mg d1-3) for two cycles on July 3, 2018 and July 31, 2018. The levels of tumor marker carcinoembryonic antigen (CEA) and carbohydrate antigen 199 (CA199) progressively declined.

From September 8, 2018, the patient was treated with immunotherapy (nivolumab [Bristol-Myers Squibb, Opdivo, Brooklyn, NY, USA] $100 \mathrm{mg} / \mathrm{m}^{2}$ ) every 2 weeks. Nivolumab was approved by the China Food and Drug Administration on June 15, 2018. Meanwhile, chemotherapy was used continually, and suspended after six cycles. Subsequently, the patient continued to receive treatment with immunotherapy. The levels of CEA, cytokeratin, and CA199 progressively decreased. In addition, the size of the masses in the lung and pelvis were also reduced (Figures 1E, F). The observed changes in tumor markers were consistent with the therapeutic efficacy and tumor load (Figure 1).

According to the follow-ups performed between October 2018 and April 2019, the patient had been able to conduct normal daily life activities, with a markedly improved quality of life and absence of additional abnormalities.

However, the patient presented with recurrent cough and hemoptysis on April 18, 2019. Lung CT imaging revealed the presence of new tissues (Figure $3 A$ ). These new tissues detected in the right main bronchus were removed using an endoscope (Figure 3B). The size of the largest new tissue was $3 \times 1 \mathrm{~cm}$, and the 
pathological diagnosis suggested hemorrhage and fibrous exudation (Figure $3 C$ ). The new tissues detected through a second respiratory endoscopy suggested the presence of malignant epithelial tumors of the salivary gland type, based on the morphology with H\&E staining, $\times 40$ (Figures $4 \mathrm{~A}$ ) and H\&E staining, $\times 100$ (Figure 4B). The immunohistochemistry results showed CD117(+/-), DOG-1(-), CK7(+), CK5/6(-), P63(-), CEA minority(+), thyroid transcription factor-1(-), Napsin A(-), CR(-), P40(-), P53 mutant, smooth muscle actin(+), S-100(-), and Ki-67(+) (approximately 50\%). The masses did not show significant change in the lung CT image (Figure 3D). These findings indicated progressive disease. After consultation with the Fudan University Shanghai Cancer Center, the use of local radiotherapy was suggested after discontinuation of immunotherapy. In June 2019, the patient received radiotherapy with DT45GY/15F for the lesions in the right lung, and mediastinal and hilar lymph nodes. The treatment alleviated the patient's cough and hemoptysis did not recur. Two weeks later, it was observed that the mass in the lung had decreased in size (Figure 3E). However, interstitial inflammation occurred after DT36Gy/12F radiotherapy (Figure 3F). Radiotherapy was discontinued and anti-infective and antiinflammatory treatments were administered. The inflammation in the lung was resolved (Figure 3G). In the end, this patient died from Recurrent pneumonia in October 2019.

\section{Discussion And Conclusion}

The treatment strategy for CUP is challenging. Poorly differentiated neuroendocrine tumors are invariably characterized by aggressive clinical behaviors, and usually associated with a poor prognosis. Typically, CUP responds to chemotherapy (cisplatin and etoposide) with $70-80 \%$ overall response rates. ${ }^{3}$ Ohta et al. found that patients with CUP who received chemotherapy showed a relatively good prognosis. ${ }^{4}$ However, chemotherapy has no impact on overall survival. Notably, gene detection can identify the source of CUP tissues, while another promising strategy involves liquid biopsies that detect circulating tumor DNA in patients with CUP. The detection of gene mutations (e.g., mismatch repair and tumor mutational burden) may help with immunotherapy, and plays an important role in the development of treatment strategies against CUP. ${ }^{4}$

Immunosuppressive agents carry considerable potential for the treatment of patients with cancer, such as advanced non-small cell lung cancer (NSCLC) and small cell lung cancer. ${ }^{5}$ Programmed cell death protein 1 (PD1) is a member of the immunoglobulin family B7-CD28. It is expressed on monocytes, $B$ cells, activated T cells, and dendritic cells as an immunosuppressive receptor. ${ }^{6}$ In healthy organisms, PD1 is mainly expressed in activated immune cells, promoting the maturation of $\mathrm{T}$ cells and regulating excessive immune responses through a negative regulatory mechanism. PD-L1 and PD-L2 are ligands for PD1. Over-activation of the PD1/PD-L1 pathway leads to inhibition of the immune response, ${ }^{7}$ thereby preventing excessive inflammatory responses. PD-L1 is the major ligand, and its expression in tumor tissues (e.g., NSCLC, breast cancer, melanoma, gastric cancer, renal cancer, etc.) is significantly higher than that recorded in normal tissues. ${ }^{8-12}$ Blockage of PD1/PD-L1 inhibits the proliferation and viability of $\mathrm{T}$ and $\mathrm{B}$ cells. It plays an important role in tumor immune escape, and promotes the progression of tumors. Overexpression of PD1 ligands in tumor cells cause weakening or even death of T cells. ${ }^{13}$ 
Therefore, blockage of PD1/PD-L1 enhances the effect of immune cells on tumor cells, and that has got benefits from clinical trials.

Nivolumab is one of the drugs which block PD1/PD-L1. In 2015, it was reviewed by the Food and Drug Administration (USA) for the treatment of previously treated advanced NSCLC. The Food and Drug Administration (USA) approved nivolumab as the immune checkpoint inhibitor for the treatment of this disease. In a phase 3 clinical trial (CheckMate-017), 51,272 patients with lung squamous cell carcinoma were randomized to receive either nivolumab or platinum and docetaxel. The results showed that nivolumab was significantly better than the docetaxel regimen in terms of objective response rate $(20 \%$ vs. $9 \%$, respectively; $P=0.008)$, and significantly prolonged progression-free survival and overall survival. Clinically, approximately $60 \%$ of patients with NSCLC have abnormal PD-L1 expression. ${ }^{14}$ However, the correlation between the expression of PD-L1 and prognosis remains unclear. It is recommended to determine the expression of PD-L1 in NSCLC patients without a driver gene mutation. Immunosuppressive therapy or combined chemotherapy with PD-1 is recommended for patients with high PD-1 expression, demonstrating the new direction toward individualized therapy for NSCLC based on gene detection. Therefore, it is emphasized that gene testing should be performed to determine the presence of gene mutations and PD-1 expression in patients prior to the administration of lung cancertargeted drugs and immune drugs. Another phase II study found that PD-L1 expression in patients with lung squamous cell carcinoma was not related to treatment efficacy. After treatment with an anti-PD-L1 agent, partial remission was achieved in $24 \%$ of PD-L1-positive patients and $14 \%$ of PD-L1-negative patients. Currently, there are many challenges in clinical practice that require urgent attention. For example, the PD-1/PD-L1 monoclonal antibody is not effective in all patients. Therefore, it is especially important to select the population that would benefit the most from this therapy. The CheckMate-227 study showed that the tumor mutational burden may be helpful in predicting the efficacy of immunotherapy. ${ }^{15}$ PD-1 immunotherapy may be appropriate for patients carrying TP53 and KRAS mutations. ${ }^{16}$ Currently, there is no gold standard for the detection of PD-L1, and it remains unclear whether PD-L1 could become an ideal molecular marker. Nivolumab was listed in China for second-line treatment of NSCLC in 2018. Therefore, there is less data in clinical practice in China. It's a new trail or challenge for this patient in our case report to accept immunotherapy.

Local radiotherapy was also used in this case, resulting in a certain therapeutic effect against CUP. In a case of primary retroperitoneal serous adenocarcinoma, the combination of immunotherapy with radiotherapy decreased the tumor size, and subsequently led to durable disease control. ${ }^{17}$ Another research study showed that radiotherapy may improve systemic responses to anti-PD1/PD-L1-directed immune therapy in Merkel cell carcinoma, as well as the immunotherapy has the clarity on abscopal regression. ${ }^{18}$ Coincidentally, a case report also suggested that immunotherapy increases the chance of an abscopal effect occurring after radiation therapy in a patient with lung adenocarcinoma treated with atezolizumab and subsequently combined with brain irradiation for the treatment of metastasis. ${ }^{19}$ However, unexpectedly, serious interstitial pneumonia in the contralateral lung field was noted in the present case, which may have been related to the long-term use of immunotherapy. Traditional radiation 
pneumonitis is usually associated with the range of exposure and dose distribution. However, in this case, the range of interstitial pneumonia was mainly observed in the contralateral lung field. This suggests that low-dose radiation may be the trigger for the activation of immune pneumonia. Alternatively, the paracrine signaling of the tumor may trigger this immune pneumonia. Radiation induces a bystander response and abscopal effects through a series of biological effects in irradiated cells, or cellular communication with non-irradiated local neighbors or distant cells. ${ }^{20}$ This has been adopted by an increasing number of scholars. However, distinguishing between radiation pneumonitis and immune checkpoint blockade-related pneumonitis remains important and challenging. ${ }^{21}$

Therefore, the combination of immunotherapy with radiotherapy requires caution, due to the risk of immune inflammation. Further research is warranted to identify optimal approaches for incorporating radiotherapy in combination treatments with respect to dosage, as well as the fractionation and sequencing of therapies. ${ }^{22}$

We reported a unique case of CUP with multiple systemic metastases in a 36-year-old male. In patients in whom the primary tumor site cannot be identified, apart from conventional chemotherapy and radiotherapy, immunotherapy can also be an effective treatment with fewer side effects and better patient tolerance. Besides, the use of radiotherapy after immunotherapy requires caution, due to the risk of immune inflammation.

\section{Abbreviations}

\begin{tabular}{|ll|}
\hline CUP & cancer of Unknown Primary \\
\hline CT & computed tomography \\
\hline CK & cytokeratin \\
\hline CDX2 & caudal-related homeodomain transcription 2 \\
\hline PD-L1 & programmed cell death 1 ligand 1 \\
\hline CEA & carcinoembryonic antigen \\
\hline CA199 & carbohydrate antigen 199 \\
\hline NSCLC & non-small cell lung cancer \\
\hline PD1 & Programmed cell death protein 1 \\
\hline
\end{tabular}

\section{Declarations}

\section{Competing Interests}

The authors declare that the research was conducted in the absence of any commercial or financial relationships that could be construed as a potential conflict of interest. 


\section{Funding}

Not Applicable

\section{Authors' Contributions}

PW and SD provided this case. QN, KL, and CP wrote, reviewed, and/or modified the manuscript. All authors have read and approved the manuscript

\section{Ethics Approval and Consent to Participate}

The study was approved by the Human Ethics Review Committee of Jiangsu Taizhou People's Hospital.

\section{Consent to Publish}

Written informed consent to publish this report and the associated medical images was provided by the patient's wife.

\section{Availability of Data and Materials}

All datasets generated for this study are included in the manuscript and/or the supplementary files.

\section{Acknowledgments}

We thank Mr. Xi Yang, PhD, from the Fudan University Shanghai Cancer Center (Shanghai, China), for his excellent assistance in editing a draft of this manuscript.

\section{References}

1. Li M, Li H, Hong G, et al. Identifying primary site of lung-limited Cancer of unknown primary based on relative gene expression orderings[J]. BMC Cancer, 2019;19:67.

2. Bochtler T, Kramer A. Does Cancer of Unknown Primary (CUP) Truly Exist as a Distinct Cancer Entity? [J]. Front Oncol, 2019;9:402.

3. Losa F, Iglesias L, Pane M, et al. 2018 consensus statement by the Spanish Society of Pathology and the Spanish Society of Medical Oncology on the diagnosis and treatment of cancer of unknown primary[J]. Clin Trans/ Oncol, 2018;20:1361-1372.

4. Ohta S, Cho Y, Shibata M, et al. Possibility of molecular targeting therapy for the treatment of cancer of unknown primary origin by analysis of intracellular signaling molecules[J]. Exp Ther Med, 2012;3:547-549.

5. Chae Y K, Pan A, Davis A A, et al. Recent Advances and Future Strategies for Immune-Checkpoint Inhibition in Small-Cell Lung Cancer[J]. Clin Lung Cancer, 2017;18:132-140.

6. Bae S B, Cho H D, Oh M H, et al. Expression of Programmed Death Receptor Ligand 1 with High Tumor-Infiltrating Lymphocytes Is Associated with Better Prognosis in Breast Cancer[J]. J Breast 
Cancer, 2016;19:242-251.

7. La-Beck N M, Jean G W, Huynh C, et al. Immune Checkpoint Inhibitors: New Insights and Current Place in Cancer Therapy[J]. Pharmacotherapy, 2015;35:963-976.

8. Schalper K A, Velcheti V, Carvajal D, et al. In situ tumor PD-L1 mRNA expression is associated with increased TILs and better outcome in breast carcinomas[J]. Clin Cancer Res, 2014;20:2773-2782.

9. Wolchok J D, Chan T A. Cancer: Antitumour immunity gets a boost[J]. Nature, 2014;515:496-498.

10. Errico A. Melanoma: CheckMate 067--frontline nivolumab improves PFS alone or in combination with ipilimumab[J]. Nat Rev Clin Oncol, 2015;12:435.

11. Maine C J, Aziz N H, Chatterjee J, et al. Programmed death ligand-1 over-expression correlates with malignancy and contributes to immune regulation in ovarian cancer[J]. Cancer Immunol Immunother, 2014;63:215-224.

12. Homet M B, Ribas A. Anti-programmed cell death protein-1/ligand-1 therapy in different cancers[J]. $\mathrm{Br}$ J Cancer, 2015;112:1421-1427.

13. He J, Hu Y, Hu M, et al. Development of PD-1/PD-L1 Pathway in Tumor Immune Microenvironment and Treatment for Non-Small Cell Lung Cancer[J]. Sci Rep, 2015;5:13110.

14. Gettinger S, Herbst R S. B7-H1/PD-1 blockade therapy in non-small cell lung cancer: current status and future direction[J]. Cancer J, 2014;20:281-289.

15. Hellmann M D, Ciuleanu T E, Pluzanski A, et al. Nivolumab plus Ipilimumab in Lung Cancer with a High Tumor Mutational Burden[J]. N Engl J Med, 2018;378:2093-2104.

16. Dong Z Y, Zhong W Z, Zhang X C, et al. Potential Predictive Value of TP53 and KRAS Mutation Status for Response to PD-1 Blockade Immunotherapy in Lung Adenocarcinoma[J]. Clin Cancer Res, 2017;23:3012-3024.

17. Chae Y K, Saleem N, Roh Y, et al. Exceptional response to chemotherapy followed by concurrent radiotherapy and immunotherapy in a male with primary retroperitoneal serous Adenocarcinoma: a case report and literature review[J]. BMC Cancer, 2019;19:748.

18. Bloom B C, Augustyn A, Pezzi T A, et al. Rescue of Immunotherapy-Refractory Metastatic Merkel Cell Carcinoma With Conventionally Fractionated Radiotherapy and Concurrent Pembrolizumab[J]. Front Oncol, 2019;9:223.

19. Lin X, Lu T, Xie Z, et al. Extracranial abscopal effect induced by combining immunotherapy with brain radiotherapy in a patient with lung adenocarcinoma: A case report and literature review[J]. Thorac Cancer, 2019;10:1272-1275.

20. Rodel F, Frey B, Multhoff G, et al. Contribution of the immune system to bystander and non-targeted effects of ionizing radiation[J]. Cancer Lett, 2015;356:105-113.

21. Schoenfeld J D, Nishino M, Severgnini M, et al. Pneumonitis resulting from radiation and immune checkpoint blockade illustrates characteristic clinical, radiologic and circulating biomarker features[J]. J Immunother Cancer, 2019;7:112. 
22. Dagoglu N, Karaman S, Caglar H B, et al. Abscopal Effect of Radiotherapy in the Immunotherapy Era: Systematic Review of Reported Cases[J]. Cureus, 2019;11:e4103.

\section{Figures}

A

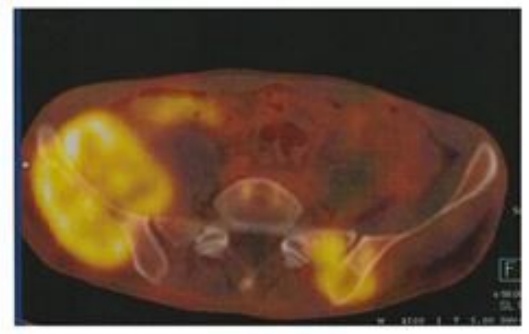

2018.04.18

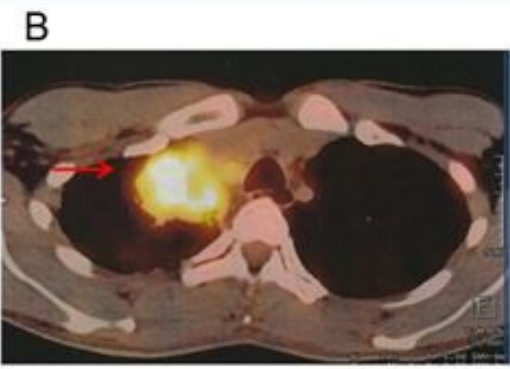

Cytokeratin 19: $3.05 \mathrm{ng} / \mathrm{mL}$ CA199: $55.23 \mathrm{U} / \mathrm{ml}$

XELOX

RT
C

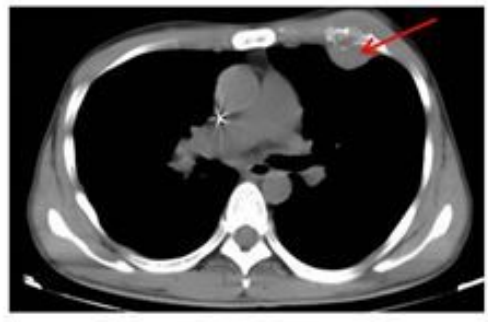

2018.07.03
E

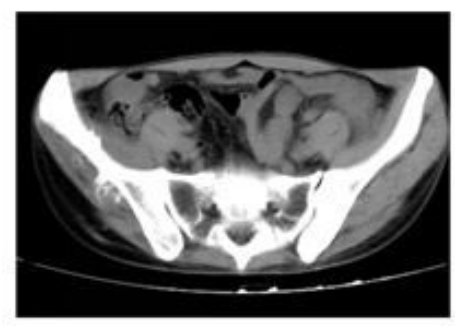

2019.04.09

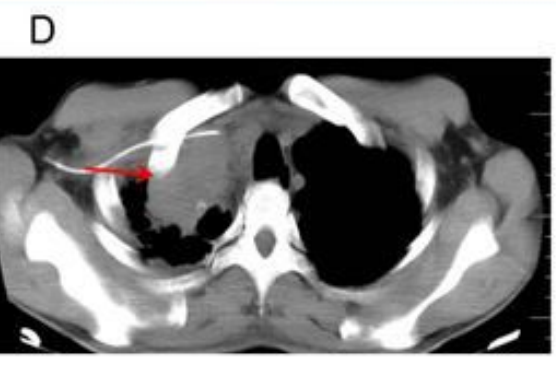

Cytokeratin 19: $8.1 \mathrm{ng} / \mathrm{mL}$ CA199: $128 \mathrm{U} / \mathrm{ml}$

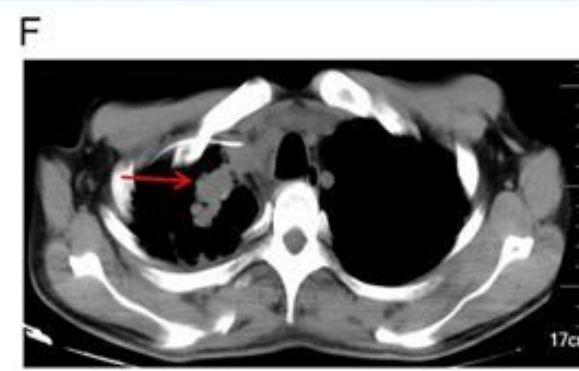

Cytokeratin 19: $0.79 \mathrm{ng} / \mathrm{mL}$ CA199: $5.19 \mathrm{U} / \mathrm{ml}$

PD1

\section{Figure 1}

Timeline of a case of CUP with multiple systemic metastases treated with a combination of immunotherapy and chemotherapy. (A) PET-CT imaging of the patient prior to any treatment, showing bone metastasis and a surrounding soft tissue mass in the pelvis. (B) The mass in the lung was a soft tissue showing a maximum cross-section of $42 \times 56 \mathrm{~mm}$. (C) The mass on the chest wall had a size of $15 \times 15 \mathrm{~mm}$. (D) Lung CT imaging of the patient after treatment with XELOX chemotherapy. The mass in the lung showed a maximum cross-section of $68 \times 44 \mathrm{~mm}$. (E) CT imaging of the pelvis for last review. (F) Lung CT imaging after chemotherapy and immunotherapy. The mass in the lung showed a maximum cross-section of $35 \times 24 \mathrm{~mm}$. CUP, cancer of unknown primary; PET-CT, positron emission tomography computed tomography 


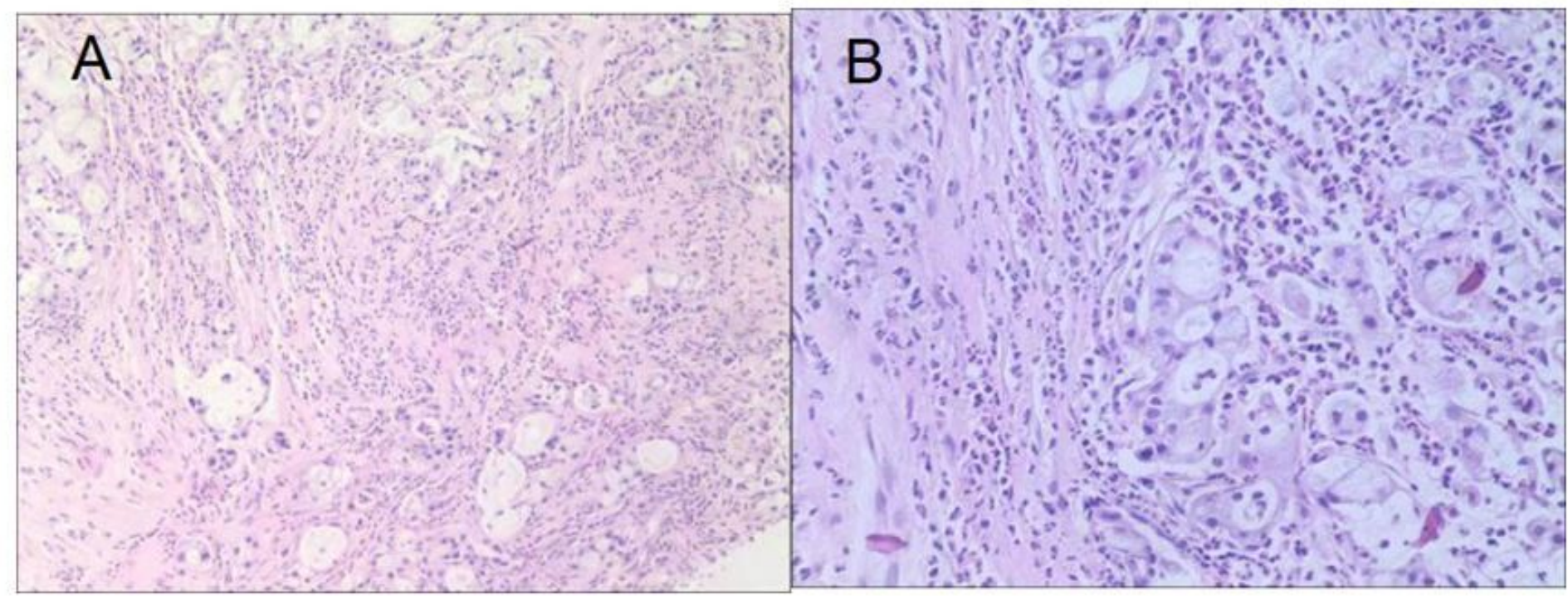

\section{Figure 2}

The mass on the chest wall was composed of poorly differentiated mucinous adenocarcinoma of the solid type. (A) H\&E staining, $\times 40$. (B) H\&E staining, $\times 100$. H\&E, hematoxylin and eosin

A

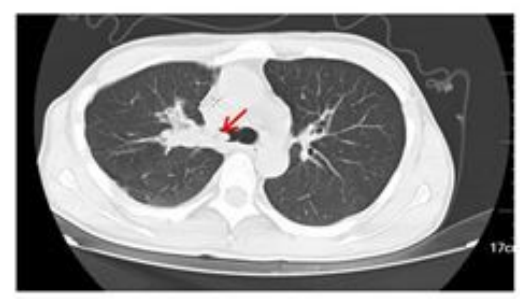

2019.04.18

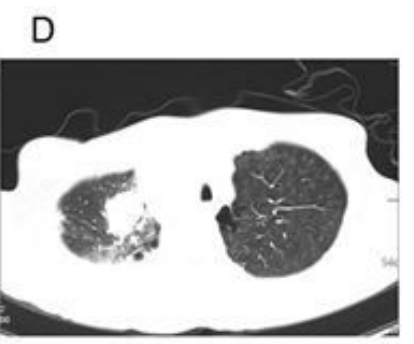

RT

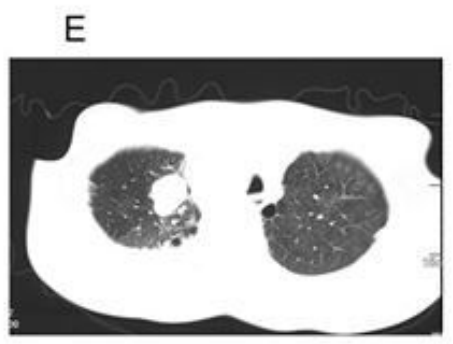

2019.06.06

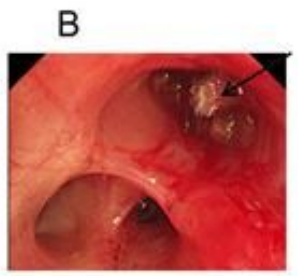

C

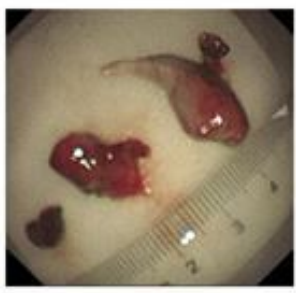

RT

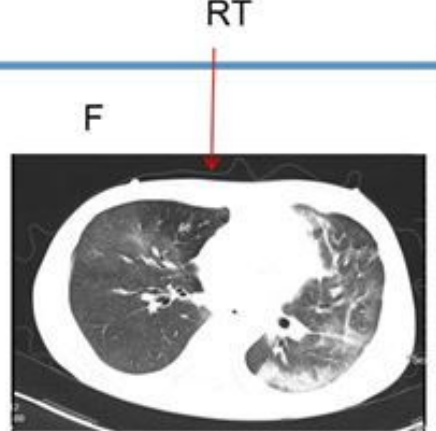

2019.06.20
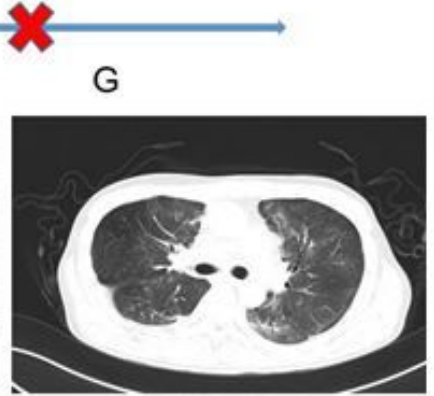

2019.08.01

\section{Figure 3}

Timeline of a case of CUP with multiple systemic metastases treated with radiotherapy after immunotherapy. (A) New tissues in the lung CT images of the patient. (B) The new tissues in the right main bronchus under the endoscope. (C) The largest new tissue in the bronchus. (D) The mass did not show significant change in the lung CT image. (E) The mass in the lung decreased in size. (F) Interstitial inflammation appeared on the CT image. (G) Interstitial inflammation in the lung was reduced. CUP, cancer of unknown primary; $\mathrm{CT}$, computed tomography 


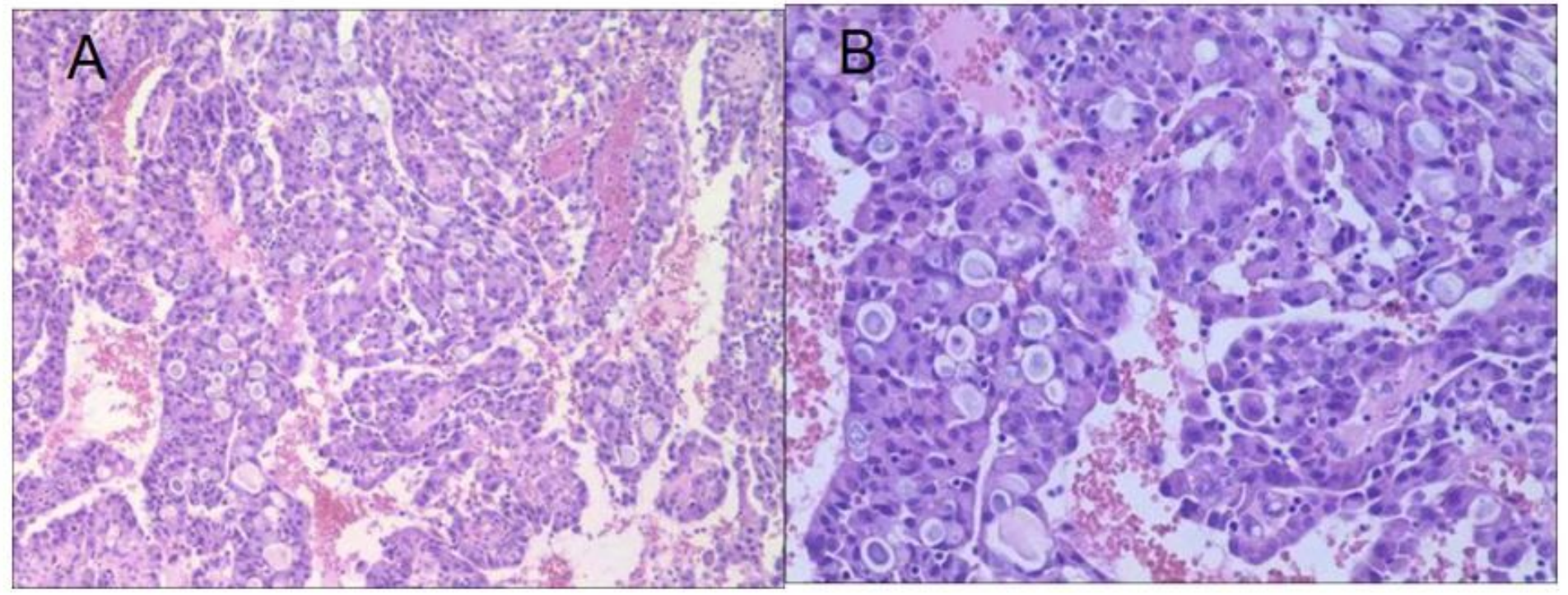

\section{Figure 4}

The new tissues detected through second respiratory endoscopy mainly comprised malignant epithelial tumors of the salivary gland type. (A) H\&E staining, $\times 40$. (B) H\&E staining, $\times 100$. H\&E, hematoxylin and eosin

\section{Supplementary Files}

This is a list of supplementary files associated with this preprint. Click to download.

- checklist.pdf 